\title{
A rare case of hepatoid adenocarcinoma in the lung and literature review: case report
}

\author{
Fudong Wang ${ }^{1}$, Yuan Weng ${ }^{1}$, Jiqun Geng ${ }^{1}$, Jun Zhu ${ }^{1}$, Baoming Mi ${ }^{2}$, Xinyi Zhou ${ }^{3}$ Jing Qian ${ }^{3}$, Qingjun You ${ }^{1}$ \\ ${ }^{1}$ Department of Thoracic Surgery, The Affiliated Hospital of Jiangnan University, Wuxi, China; ${ }^{2}$ Department of PET-CT center, The Affiliated \\ Hospital of Jiangnan University, Wuxi, China; ${ }^{3}$ Department of Pathology, The Affiliated Hospital of Jiangnan University, Wuxi, China \\ Correspondence to: Qingjun You. Department of Thoracic Surgery, The Affiliated Hospital of Jiangnan University, 200 Huihe Road, Binhu, Wuxi \\ 214000, China. Email: wxsytsg@126.com.
}

\begin{abstract}
Hepatoid adenocarcinoma (HAC) is a rare type of adenocarcinoma with adenoid and hepatocytelike differentiation that occurs outside the liver. It is highly malignant and has poor prognosis. The tumor can occur in different organs, most often being in the stomach. Primary hepatoid adenocarcinoma of the lung (HAL) are extremely rare with few reports. Here, we report a case of a 67-year-old male with HAL. Serum alpha-fetoprotein (AFP) level at diagnosis was $>2,000 \mathrm{ng} / \mathrm{mL}$. He underwent right lower lobectomy with lymph nodes dissection under video-assisted thoracoscopic surgery (VATS) followed by adjuvant chemotherapy (Docetaxel $100 \mathrm{mg}$ D1 + Nedaplatin $100 \mathrm{mg}$ D2 for six sessions). Two months after surgery, the level of serum AFP declined to normal level. The patient received regular outpatient follow-up and no local recurrence or distant metastasis was found after 18 months and the serum AFP level remained within the normal range. Review of the literature showed that HAL is a rare highly malignant disease with no standard treatment scheme. We need more cases and further studies to explore for treatment of this rare disease.
\end{abstract}

Keywords: Lung neoplasm; hepatoid adenocarcinoma (HAC); immunohistochemistry; case report

Received: 26 November 2019; Accepted: 10 February 2020; Published: 25 August 2020.

doi: $10.21037 /$ ccts.2020.02.04

View this article at: http://dx.doi.org/10.21037/ccts.2020.02.04

\section{Introduction}

Hepatoid adenocarcinoma of the lung (HAL) is an extremely rare malignant tumor originating from the lung with both hepatocellular carcinoma and adenocarcinomalike characteristics. The tumor cells can secrete molecules such as alpha-fetoprotein (AFP), HepPar1, HEA125 and MOC31. This disease occurs mostly in patients over 50 -year-old and is more common in men who have a smoking history (1). Usually, there is no specific clinical symptoms, most patients are not diagnosed until advanced stage and have poor prognosis. Because of the extremely low incidence and strong tumor heterogeneity, so far there is still no uniform treatment guideline of HAL. We are reporting a case of HAL, and also review the literature to further study the clinicopathological features, immunophenotype, diagnosis, treatment and prognosis of this disease. We present the following case in accordance with the CARE Guideline (2).

\section{Case presentation}

The patient was a 67 -year-old man who presented with a right lower lobe lung mass. He had no symptoms such as cough, chest discomfort, chest pain or hemoptysis. He had a history of hypertension for more than 10 years, but no history of smoking, hepatitis, or other tumor. No positive signs were found in physical examination. Computed tomography (CT) examination showed an irregular $4.1 \mathrm{~cm} \times 3.5 \mathrm{~cm}$ mass in the right lower lobe with uneven enhancement and faint edge. There was bilateral pleural thickening and the hilar and mediastinal lymph nodes were not enlarged. Abdominal CT showed no tumor in the liver (Figure 1A). Emission computerized tomography (ECT) showed no evidence of bone metastasis (Figure 1B). Positron 
A
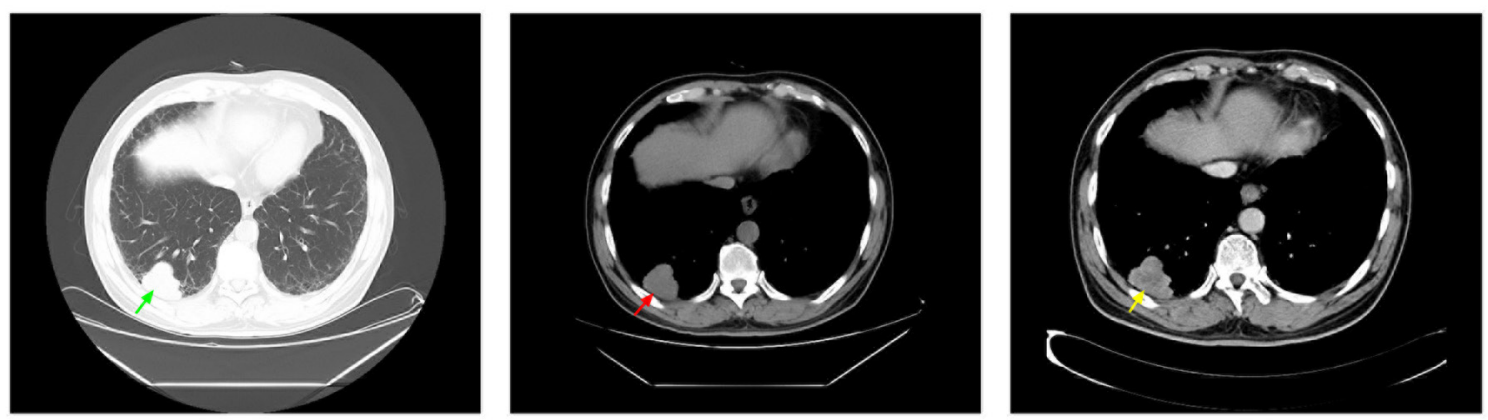

B

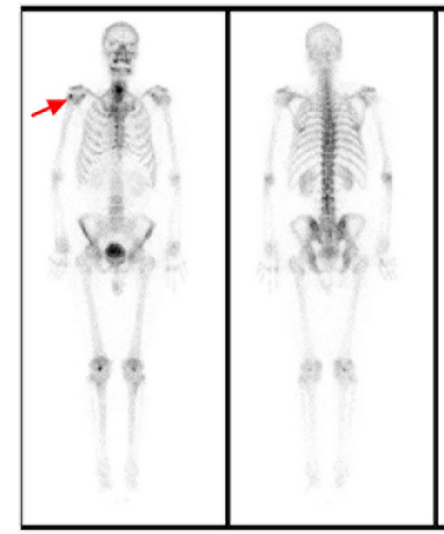

C

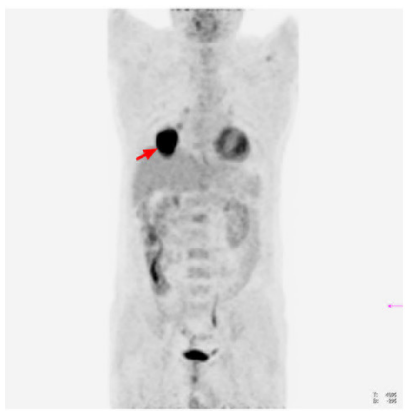

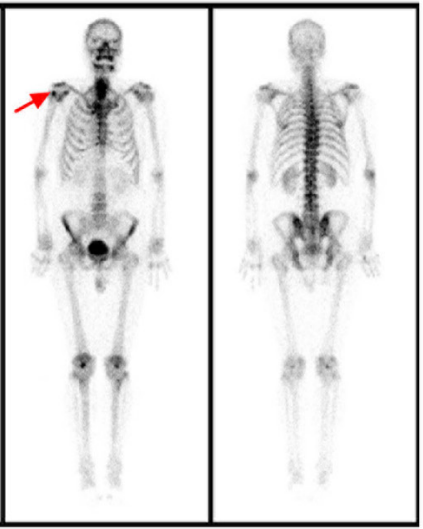
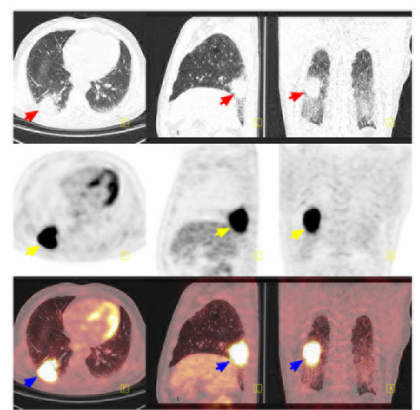

$D$

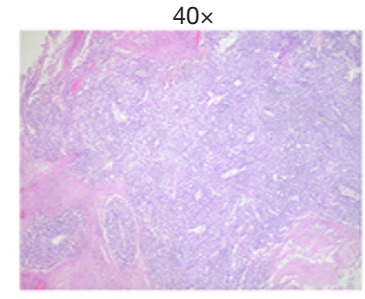

AFP

\section{E}

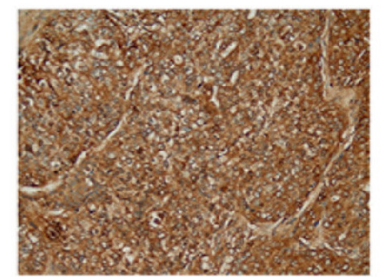

HepPar1

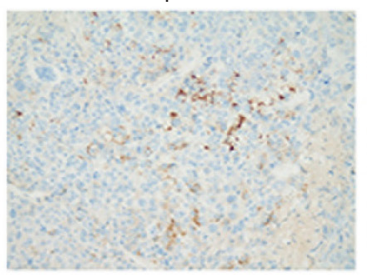

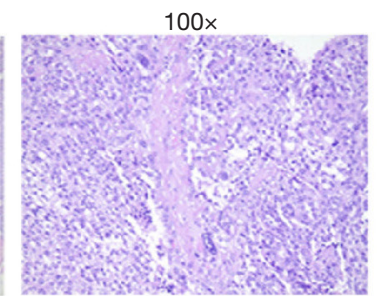

CDX-2

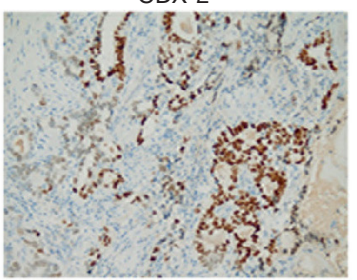

CK7

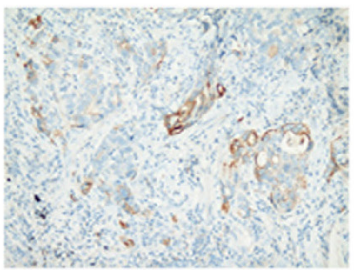

Figure $1 \mathrm{CT}$ of the patient (A) (left, lung window; middle, mediastinal window) showed a soft tissue mass (green and red arrowhead) with its size about $4.1 \mathrm{~cm} \times 3.5 \mathrm{~cm}$ in the right lower lobe. The lesion (yellow arrowhead) showed mild heterogeneous enhancement on the contrast CT image (right). ECT of the patient (B) showed the metacarpal osteogenesis of the right humeral head is increased (red arrowhead), and it is recommended to follow up. There is no obvious abnormality in the remaining bone imaging. PET-CT of the patient (C) showed there is abnormal FDG metabolism in the right lower lobe mass, SUVmax =19.02 (left panel, red arrowhead), the right panel showed CT (red arrowhead), PET (yellow arrowhead) and PET-CT (blue arrowhead) of the tumor; no significant abnormal increase in metabolism was observed in FDG; no significant abnormal increase in FDG metabolism was observed in PET imaging of the whole body. Pathology of hepatoid adenocarcinoma of the lung (D) showed poorly differentiated adenocarcinoma with local necrosis (HE staining, left: 40x; right: 100x). Immunohistochemistry results (E) of HAL (upper left: AFP positive; upper right: CDX-2 partial positive; lower left: HepPar1 small positive; lower right: CK7 small positive). CT, computed tomography; ECT, emission computerized tomography; PET, positron emission tomography; FDG, fluorodeoxyglucose; AFP, alpha-fetoprotein.

emission tomography (PET)-CT showed abnormal fluorodeoxyglucose (FDG) metabolism in the right lower lobe mass (SUVmax $=19.02)$. The FDG metabolism of right hilar lymph nodes was increased (SUVmax $=3.82$ ) considering the possibility of metastasis. The mediastinal lymph nodes and the rest of the body showed no significant 
abnormal increase in FDG (Figure $1 C$ ). Laboratory examination: AFP: >2,000 ng/mL, CY211: $4.63 \mathrm{ng} / \mathrm{mL}$, liver function was normal.

The patient underwent video-assisted thoracoscopic surgery (VATS) right lower lobe resection and lymph nodes dissection (stations 2, 7, 10, 11) on 06-27-2018. Postoperative pathology demonstrated a $5.5 \mathrm{~cm} \times 4.0 \mathrm{~cm}$ $\times 3.8 \mathrm{~cm}$ poorly differentiated adenocarcinoma of the right lower lobe with local necrosis but no lymph node metastasis. There was no vascular tumor thrombus and no invasion of the nerve. The bronchial margin was negative, and the pleural surface of the tumor was close but not involved by the cancer (Figure 1D). Immunohistochemistry results: $\operatorname{AFP}(+), \operatorname{CDX}-2($ partial +$)$, CK7 (small +), CK20(individual +), HepPar1(small +), ALK(-), P40(-), PE10/SPA(-), NapsinA(-), CK5/6(-), Syn(-), CgA(-), PLAP(-), CD30(-), CD56(-), CD117(-), Vimentin(-), P63(-), TTF-1(-), considering poorly differentiated cancer with hepatic differentiation (Figure $1 E$ ). The patient was discharged a week after surgery with no complications. $\mathrm{He}$ received adjuvant chemotherapy (Docetaxel $100 \mathrm{mg} \mathrm{D1}$ + Nedaplatin 100 mg D2 for six sessions) with no adverse events. Two months after surgery, the level of serum AFP declined to normal. The patient received regular outpatient follow-up and no local recurrence or distant metastasis was found after 18 months and the serum AFP level remained within the normal range.

All procedures performed in this study were in accordance with the ethical standards of the institutional and national research committees and with the Helsinki Declaration (as revised in 2013). Written informed consent was obtained from the patient for publication of this case report and accompanying images. A copy of the written consent is available for review by the editorial office of this journal.

\section{Discussion}

HAC is a rare malignant liver-like tumor that occurs outside the liver. It is similar to hepatocellular carcinoma in morphology and has adenocarcinoma-like characteristics. The tumor can occur in various organs. Metzgeroth et al. reviewed the clinical features of 261 cases of HAC and found that the most common site of HAC was stomach (63\%), and other sites included ovary (10\%), lung (5\%), gallbladder (4\%), pancreas (4\%), and uterus (4\%) (3). Primary HAL are extremely rare and the origin of HAL remains unclear. Ishikura et al. thought that HAL most likely originate from ectopic hepatocytes or germ cells in the lung tissue and respiratory epithelium during embryonic development (1). Some lung cancer cells may differentiate into hepatocytes which then develop into HAL. Saka et al. considered this disease to be an intrapulmonary manifestation of extragonadal germ cell cancer syndromes (4). Sinard et al. believed that hepatocytes have abnormal differentiation in other parts of the body outside the liver leading to HAC (5).

HAL usually occurs at the older age (over 50 years old) male with history of smoking. Our case presented here was an elderly male patient with no history of smoking. Most of the patients with HAL were in advanced stage with elevated serum AFP levels (Appendix 1). In this case, the preoperative AFP level was $>2,000 \mathrm{ng} / \mathrm{mL}$ and declined to $1,180.12 \mathrm{ng} / \mathrm{mL}$ 1 week after surgery. Two months later, the AFP level became normal. The cut surface of the gross specimen was gray or gray-brown, with clear boundaries. The central necrosis composed of hepatic-like differentiation zone and non-hepatic-like differentiation zone, and the hepatic-like differentiation zone cancer tissue was nested and beam-like. The cancer cells were large, polygonal, rich in cytoplasm, eosinophilic or transparent, with PAS-positive small body. The nucleus was large, dark staining and in abnormal shape. The interstitium could appear more sinusoids and often have necrosis. Non-hepatic-like differentiation areas could be seen in adenoid and papillary structures, and a few areas were poorly differentiated adenocarcinoma-like changes.

Ishikura et al. reported in 1990 that HAC can be diagnosed when a typical alveolar or papillary adenocarcinoma and a component similar to hepatocellular carcinoma are observed and AFP is expressed (1). However, with the increased number of cases reported, we found that AFP is not always expressed in HAC. Therefore, it is necessary to use other specific molecules for immunohistochemical staining to assist diagnosis. HepPar1 is a relatively specific hepatocyte marker, and it has no obvious crossover with other tumors. Immunostaining of CK molecules also contributes to the diagnosis of HAC. Study reported that CK18 is positive in HAL, while CK20 is negative, and CK7 staining may be positive or negative (6). In this case, $\mathrm{AFP}(+)$, CDX$2($ partial + ), CK7 (small amount + ), CK20(individual + ), and HepPar1(small amount + ) were observed, and were similar to those reported in the literature. In summary, we believe that the diagnosis of HAL should mainly rely on the morphological similarity to hepatocellular carcinoma, combined with immunohistochemical markers AFP, hepatocyte, CK18 and other positively expressed molecules, 
and excluded by clinical pathology and immunohistochemistry metastasis of diseases such as hepatocellular carcinoma.

So far, there is no standard treatment scheme for HAL. Surgical resection, adjuvant chemotherapy and radiotherapy are common choices for the treatment of this disease (7). In recent years, molecular targeted therapy has also been shown to have some curative effect (Appendix 1). Khozin et al. reported the first case of treatment of HAL with the tyrosine kinase inhibitor crizotinib, resulting in a reduction in tumor volume (8). Gavrancic et al. recently reported the use of platinum combined with sorafenib in the treatment of a patient with stage IV HAL and achieved partial remission with stability lasting for 11 months, this was the longest survival for patients with stage IV HAL (9). Basse $e t$ al. reported that immunotherapy was used for HAL, although programmed death ligand 1 (PD-L1) was negative in this patient's tumor cells and immune cells. The patient did have a partial response to immunotherapy, but unfortunately, the patient eventually died of infection (10).

Previous studies have shown that the prognosis of HAL is poorer than other types of lung tumors. However, if the disease is found early and the tumor is small, the prognosis could be good after complete surgical resection (9). In our case, the tumor stage was (pT3N0M0, IIB), with no lymph nodes metastasis, no vascular and neural invasion, but the degree of tumor differentiation was poor. After lobectomy with adjuvant chemotherapy, there was no local recurrence or distant metastasis and the serum AFP level remained within the normal range 18 months after surgery.

Our case report has some strengths as follows: on one thing, our patient has survived with no disease relapse or progress for 18 months, this is a pleased prognosis; on another thing, the pleased prognosis may owe to our adjuvant chemotherapy formula. However, there is limitations in our report, we need perform regular outpatient follow-up and present the natural course of HAL

\section{Conclusions}

HAL is a rare malignant tumor originating from the lung with hepatocellular carcinoma and adenocarcinoma-like characteristics and has a poor prognosis. The diagnosis of HAL should mainly rely on the morphological characteristics combined with immunohistochemical markers. There is no standard treatment scheme of HAL. Early diagnosis and aggressive treatment can result in long-term survival. Nevertheless, more case reports and further studies are required to explore for treatment of this rare disease.

\section{Acknowledgments}

Funding: None.

\section{Footnote}

Conflicts of Interest: All authors have completed the ICMJE uniform disclosure form (available at https://ccts. amegroups.com/article/view/10.21037/ccts.2020.02.04/ coif). The authors have no conflicts of interest to declare.

Ethical Statement: The authors are accountable for all aspects of the work in ensuring that questions related to the accuracy or integrity of any part of the work are appropriately investigated and resolved. All procedures performed in this study were in accordance with the ethical standards of the institutional and national research committees and with the Helsinki Declaration (as revised in 2013). Written informed consent was obtained from the patient for publication of this case report and accompanying images. A copy of the written consent is available for review by the editorial office of this journal.

Open Access Statement: This is an Open Access article distributed in accordance with the Creative Commons Attribution-NonCommercial-NoDerivs 4.0 International License (CC BY-NC-ND 4.0), which permits the noncommercial replication and distribution of the article with the strict proviso that no changes or edits are made and the original work is properly cited (including links to both the formal publication through the relevant DOI and the license). See: https://creativecommons.org/licenses/by-nc-nd/4.0/.

\section{References}

1. Ishikura H, Kanda M, Ito M, et al. Hepatoid adenocarcinoma: a distinctive histological subtype of alpha-fetoprotein-producing lung carcinoma. Virchows Arch A Pathol Anat Histopathol 1990;417:73-80.

2. Riley DS, Barber MS, Kienle GS, et al. CARE guidelines for case reports: explanation and elaboration document. J Clin Epidemiol 2017;89:218-35.

3. Metzgeroth G, Ströbel P, Baumbusch T, et al. Hepatoid adenocarcinoma - review of the literature illustrated by a rare case originating in the peritoneal cavity. Onkologie 2010;33:263-9.

4. Saka H, Sakai S, Kondo N, et al. Successful resection of alpha-fetoprotein-producing lung cancer. Chest 
1988;94:879-80.

5. Sinard J, Macleay L Jr, Melamed J. Hepatoid adenocarcinoma in the urinary bladder. Unusual localization of a newly recognized tumor type. Cancer 1994;73:1919-25.

6. Tsung JS, Yang PS. Hepatoid carcinoma of the ovary: characteristics of its immunoreactivity. A case report. Eur J Gynaecol Oncol 2004;25:745-8.

7. Che YQ, Wang S, Luo Y, et al. Hepatoid adenocarcinoma of the lung: Presenting mediastinal metastasis without transfer to the liver. Oncol Lett 2014;8:105-10.

doi: $10.21037 /$ ccts.2020.02.04

Cite this article as: Wang F, Weng Y, Geng J, Zhu J, Mi B, Zhou X, Qian J, You Q. A rare case of hepatoid adenocarcinoma in the lung and literature review: case report. Curr Chall Thorac Surg 2020;2:30.
8. Khozin S, Roth MJ, Rajan A, et al. Hepatoid carcinoma of the lung with anaplastic lymphoma kinase gene rearrangement. J Thorac Oncol 2012;7:e29-31.

9. Gavrancic T, Park YH. A novel approach using sorafenib in alpha fetoprotein-producing hepatoid adenocarcinoma of the lung. J Natl Compr Canc Netw 2015;13:387-91; quiz 391.

10. Basse V, Schick U, Guéguen P, et al. A Mismatch RepairDeficient Hepatoid Adenocarcinoma of the Lung Responding to Anti-PD-L1 Durvalumab Therapy Despite no PD-L1 Expression. J Thorac Oncol 2018;13:e120-2. 


\section{Supplementary: Discussion}

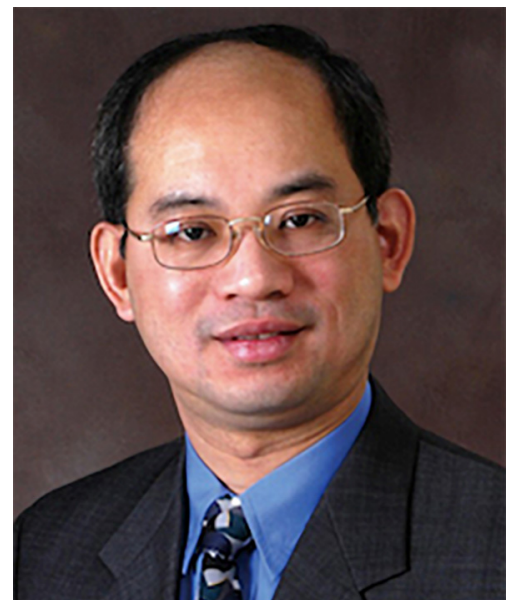

Dr. Hon Chi Suen (Hong Kong, China): Dr. Wang and colleagues reported a rare lung tumor hepatoid adenocarcinoma and reviewed the literature on this unusual pathology. Could you tell us if there are any features before surgery that may lead the physician to suspect this rare disease?

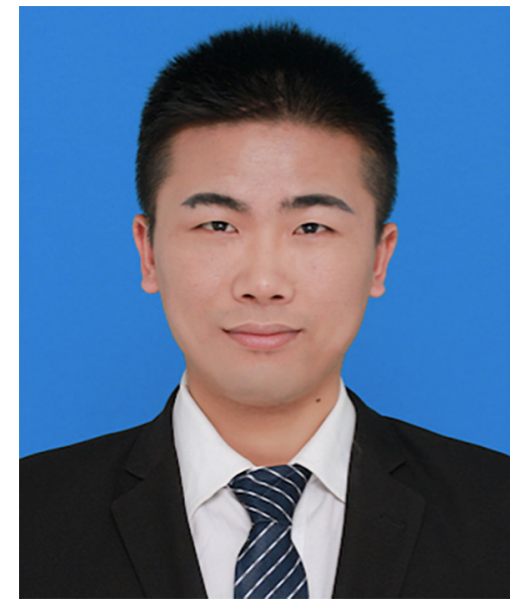

Dr. Fudong Wang (Wuxi, Jiangsu, China): HAL usually occurs at the older age male with history of smoking and most of the patients with elevated serum AFP levels. Therefore, for elderly patients with isolated lung occupation and elevated serum AFP levels, the possibility of HAL should be highly guarded after liver occupation is excluded.

\section{Dr. Suen: Are there any molecular studies that can help find good targeted therapy for this tumor that has poor prognosis?}

Dr. Wang: Molecular targeted therapy has been shown to have some curative effect. Khozin et al. reported the first case of treatment of HAL with the tyrosine kinase inhibitor crizotinib, resulting in a reduction in tumor volume. Gavrancic et al. recently reported the use of platinum combined with sorafenib in the treatment of a patient with stage IV HAL and achieved partial remission with stability lasting for 11 months. Basse et al. reported that immunotherapy was used for HAL, the patient did have a partial response to immunotherapy. We believe that with the deepening of molecular mechanism research, the therapeutic targets of HAL will increase and eventually improve the prognosis of patients. 